\title{
On Strongly Irregular Points of a Brouwer Homeomorphism Embeddable in a Flow
}

\author{
Zbigniew Leśniak \\ Institute of Mathematics, Pedagogical University, Podchorążych 2, 30-084 Kraków, Poland \\ Correspondence should be addressed to Zbigniew Leśniak; zlesniak@up.krakow.pl
}

Received 25 February 2014; Accepted 25 July 2014; Published 16 October 2014

Academic Editor: Douglas R. Anderson

Copyright (C) 2014 Zbigniew Leśniak. This is an open access article distributed under the Creative Commons Attribution License, which permits unrestricted use, distribution, and reproduction in any medium, provided the original work is properly cited.

We study the set of all strongly irregular points of a Brouwer homeomorphism $f$ which is embeddable in a flow. We prove that this set is equal to the first prolongational limit set of any flow containing $f$. We also give a sufficient condition for a class of flows of Brouwer homeomorphisms to be topologically conjugate.

\section{Introduction}

In this part we recall the requisite definitions and results concerning Brouwer homeomorphisms and flows of such homeomorphisms.

By a Brouwer homeomorphism we mean an orientation preserving homeomorphism of the plane onto itself which has no fixed points. By a flow we mean a group of homeomorphisms of the plane onto itself $\left\{f^{t}: t \in \mathbb{R}\right\}$ under the operation of composition which satisfies the following conditions:

(1) the function $F: \mathbb{R}^{2} \times \mathbb{R} \rightarrow \mathbb{R}^{2}, F(x, t)=f^{t}(x)$ is continuous,

(2) $f^{0}(x)=x$ for $x \in \mathbb{R}^{2}$,

(3) $f^{t}\left(f^{s}(x)\right)=f^{t+s}(x)$ for $x \in \mathbb{R}^{2}, t, s \in \mathbb{R}$.

We say that a Brouwer homeomorphism $f$ is embeddable in a flow if there exists a flow $\left\{f^{t}: t \in \mathbb{R}\right\}$ such that $f=f^{1}$. Then for each $t \in \mathbb{R} \backslash\{0\}, f^{t}$ is a Brouwer homeomorphism.

For any sequence of subsets $\left(A_{n}\right)_{n \in \mathbb{Z}_{+}}$of the plane we define limes superior $\lim _{\sup _{n \rightarrow \infty}} A_{n}$ as the set of all points $p \in \mathbb{R}^{2}$ such that any neighbourhood of $p$ has common points with infinitely many elements of the sequence $\left(A_{n}\right)_{n \in \mathbb{Z}_{+}}$. For any subset $B$ of the plane we define the positive limit set $\omega_{f}(B)$ as the limes superior of the sequence of its iterates $\left(f^{n}(B)\right)_{n \in \mathbb{Z}_{+}}$and negative limit set $\alpha_{f}(B)$ as the limes superior of the sequence $\left(f^{-n}(B)\right)_{n \in \mathbb{Z}}$. Under the assumption that $B$ is compact, Nakayama [1] proved that

$$
\begin{aligned}
& \begin{array}{l}
\omega_{f}(B) \\
=\left\{q \in \mathbb{R}^{2} \text { : there exist sequences }\left(p_{j}\right)_{j \in \mathbb{Z}_{+}},\right. \\
\quad\left(n_{j}\right)_{j \in \mathbb{Z}_{+}} \text {such that } p_{j} \in B, n_{j} \in \mathbb{Z}_{+}, n_{j} \longrightarrow+\infty, \\
\left.\quad f^{n_{j}}\left(p_{j}\right) \longrightarrow q \text { as } j \longrightarrow+\infty\right\}, \\
\alpha_{f}(B) \\
=\left\{q \in \mathbb{R}^{2} \text { : there exist sequences }\left(p_{j}\right)_{j \in \mathbb{Z}_{+}},\right. \\
\quad\left(n_{j}\right)_{j \in \mathbb{Z}_{+}} \text {such that } p_{j} \in B, n_{j} \in \mathbb{Z}_{+}, n_{j} \longrightarrow+\infty, \\
\left.\quad f^{-n_{j}}\left(p_{j}\right) \longrightarrow q \text { as } j \longrightarrow+\infty\right\} .
\end{array}
\end{aligned}
$$

A point $p$ is called positively irregular if $\omega_{f}(B) \neq \emptyset$ for each Jordan domain $B$ containing $p$ in its interior and negatively irregular if $\alpha_{f}(B) \neq \emptyset$ for each Jordan domain $B$ containing $p$ in its interior, where by a Jordan domain we mean the union of a Jordan curve $J$ and the Jordan region determined by $J$ (i.e., the bounded component of $\mathbb{R}^{2} \backslash J$ ). A point which is not positively irregular is said to be positively regular. Similarly, a point which is not negatively irregular is called negatively 
regular. A point which is positively or negatively irregular is called irregular, otherwise it is regular.

We say that a set $U \subset \mathbb{R}^{2}$ is invariant under $f$ if $f(U)=U$. An invariant simply connected region $U \subset \mathbb{R}^{2}$ is said to be parallelizable if there exists a homeomorphism $\varphi_{U}$ mapping $U$ onto $\mathbb{R}^{2}$ such that

$$
f(x)=\varphi_{U}^{-1}\left(\varphi_{U}(x)+(1,0)\right) \quad \text { for } x \in U
$$

The homeomorphism $\varphi_{U}$ occurring in this equality is called a parallelizing homeomorphism of $\left.f\right|_{U}$. On account of the Brouwer Translation Theorem, for each $p \in \mathbb{R}^{2}$ there exists a parallelizable region $U$ containing $p$ (see [2]).

Homma and Terasaka [3] proved a theorem describing the structure of an arbitrary Brouwer homeomorphism. The theorem can be formulated in the following way.

Theorem 1 (see [3], First Structure Theorem). Let $f$ be a Brouwer homeomorphism. Then the plane is divided into at most three kinds of pairwise disjoint sets: $\left\{O_{i}: i \in I\right\}$, where $I=$ $\mathbb{Z}_{+}$or $I=\{1, \ldots, n\}$ for a positive integer $n,\left\{O_{i}^{\prime}: i \in \mathbb{Z}_{+}\right\}$, and $F$. The sets $\left\{O_{i}: i \in I\right\}$ and $\left\{O_{i}^{\prime}: i \in \mathbb{Z}_{+}\right\}$are the components of the set of all regular points such that each $\mathrm{O}_{i}$ is a parallelizable unbounded simply connected region and each $O_{i}^{\prime}$ is a simply connected region satisfying the condition $O_{i}^{\prime} \cap f^{n}\left(O_{i}^{\prime}\right)=\emptyset$ for $n \in \mathbb{Z} \backslash\{0\}$. The set $F$ is invariant and closed and consists of all irregular points.

For an irregular point $p$ of a Brouwer homeomorphism $f$ the set $P^{+}(p)$ is defined as the intersection of all $\omega_{f}(B)$ and the set $P^{-}(p)$ as the intersection of all $\alpha_{f}(B)$, where $B$ is a Jordan domain containing $p$ in its interior. An irregular point $p$ is strongly positively irregular if $P^{+}(p) \neq \emptyset$, otherwise it is weakly positively irregular. Similarly, $p$ is strongly negatively irregular if $P^{-}(p) \neq \emptyset$, otherwise it is weakly negatively irregular. We say that $p$ is strongly irregular if it is strongly positively irregular or strongly negatively irregular. Otherwise, an irregular point $p$ is said to be weakly irregular.

Homma and Terasaka [3] proved that for all $p, q \in \mathbb{R}^{2}$

$$
q \in P^{+}(p) \Longleftrightarrow p \in P^{-}(q) \text {. }
$$

Nakayama [4] showed that for any Brouwer homeomorphism the set of strongly irregular points has no interior points. The set of weakly irregular points consists of all cluster points of the set of strongly irregular points which are not strongly irregular points (see [3]).

A counterpart of Theorem 1 for a Brouwer homeomorphism embeddable in a flow has been given in [5]. Namely, if a Brouwer homeomorphism is embeddable in a flow, then the set of regular points is a union of pairwise disjoint parallelizable unbounded simply connected regions.

\section{Strongly Irregular Points}

In this section we study the structure of the set of all irregular points for Brouwer homeomorphisms embeddable in a flow.

Let $f$ be a Brouwer homeomorphism. Assume that there exists a flow $\left\{f^{t}: t \in \mathbb{R}\right\}$ such that $f^{1}=f$. Let $U \subset \mathbb{R}^{2}$ be a simply connected region such that $f^{t}(U)=U$ for $t \in \mathbb{R}$. We say that $U$ is a parallelizable region of the flow if there exists a homeomorphism $\varphi_{U}$ mapping $U$ onto $\mathbb{R}^{2}$ such that

$$
f^{t}(x)=\varphi_{U}^{-1}\left(\varphi_{U}(x)+(t, 0)\right) \quad \text { for } x \in U, t \in \mathbb{R} .
$$

Such a homeomorphism $\varphi_{U}$ will be called a parallelizing homeomorphism of the flow $\left\{\left.f^{t}\right|_{U}: t \in \mathbb{R}\right\}$. It is known that for any simply connected region $U$ which is invariant under the flow $\left\{f^{t}: t \in \mathbb{R}\right\}$ the existence of a parallelizing homeomorphism of $\left.f\right|_{U}$ is equivalent to the existence of a parallelizing homeomorphism of $\left\{\left.f^{t}\right|_{U}: t \in \mathbb{R}\right\}$ (see [6]).

By the trajectory of a point $p \in \mathbb{R}^{2}$ we mean the set $C_{p}:=\left\{f^{t}(p): t \in \mathbb{R}\right\}$. It is known that a region $U$ is parallelizable if and only if there exists a topological line $K$ in $U$ (i.e., a homeomorphic image of a straight line that is a closed set in $U$ ) such that $K$ has exactly one common point with every trajectory of $\left\{f^{t}: t \in \mathbb{R}\right\}$ contained in $U$ (see [7], page 49). Such a set $K$ we will call a section in $U$ (or a local section of $\left\{f^{t}: t \in \mathbb{R}\right\}$ ). On account of the Whitney-Bebutov Theorem (see [7], page 52), for each $p \in \mathbb{R}^{2}$ there exists a parallelizable region $U_{p}$ containing $p$. Without loss of generality we can assume that the parallelizing homeomorphism $\varphi_{U_{p}}$ satisfies the condition $\varphi_{U_{p}}(p)=(0,0)$. Then $K_{\varphi_{U_{p}}}:=\varphi_{U_{p}}^{-1}(\{0\} \times \mathbb{R})$ is a local section containing $p$.

For a flow $\left\{f^{t}: t \in \mathbb{R}\right\}$ and a point $p \in \mathbb{R}^{2}$ we define the first positive prolongational limit set and the first negative prolongational limit set of $p$ by

$$
\begin{gathered}
J^{+}(p):=\left\{q \in \mathbb{R}^{2}: \text { there exist sequences }\left(p_{n}\right)_{n \in \mathbb{Z}_{+}},\right. \\
\left(t_{n}\right)_{n \in \mathbb{Z}_{+}} \text {such that } p_{n} \longrightarrow p, t_{n} \longrightarrow+\infty, \\
\left.f^{t_{n}}\left(p_{n}\right) \longrightarrow q \text { as } n \longrightarrow+\infty\right\}, \\
J^{-}(p):=\left\{q \in \mathbb{R}^{2}: \text { there exist sequences }\left(p_{n}\right)_{n \in \mathbb{Z}_{+}},\right. \\
\left(t_{n}\right)_{n \in \mathbb{Z}_{+}} \text {such that } p_{n} \longrightarrow p, t_{n} \longrightarrow-\infty, \\
\left.f^{t_{n}}\left(p_{n}\right) \longrightarrow q \text { as } n \longrightarrow+\infty\right\} .
\end{gathered}
$$

The set $J(p):=J^{+}(p) \cup J^{-}(p)$ is called the first prolongational limit set of $p$. For a subset $H \subset \mathbb{R}^{2}$ we define

$$
J(H):=\bigcup_{p \in H} J(p)
$$

The set $J\left(\mathbb{R}^{2}\right)$ will be called the first prolongational limit set of the flow $\left\{f^{t}: t \in \mathbb{R}\right\}$. For all $p, q \in \mathbb{R}^{2}$ we have

$$
q \in J^{+}(p) \Longleftrightarrow p \in J^{-}(q) \text {. }
$$

In [5] it has been proven that for each point $p \in \mathbb{R}^{2}$ the set $P^{+}(p)$ is contained in $J^{+}(p)$. Now we prove the converse inclusion.

Theorem 2. Let $f$ be a Brouwer homeomorphism which is embeddable in a flow $\left\{f^{t}: t \in \mathbb{R}\right\}$ and let $p \in \mathbb{R}^{2}$. Then $J^{+}(p) \subset P^{+}(p)$. 
Proof. Let $q \in J^{+}(p)$. Denote by $S_{p q}$ the strip between trajectories $C_{p}$ and $C_{q}$ of points $p$ and $q$, respectively. Then for each $z \in S_{p q}$ the trajectory $C_{p}$ is contained in the strip $S_{q z}$ between trajectories $C_{q}$ and $C_{z}$ of points $q$ and $z$, respectively, and the trajectories $C_{q}$ and $C_{z}$ are subsets of the same component of $S_{q z} \backslash C_{p}$ (see [8]). Let $K_{0}$ and $L_{0}$ be local sections of $\left\{f^{t}: t \in \mathbb{R}\right\}$ such that $p \in K_{0}$ and $q \in L_{0}$.

Let $B$ be a Jordan domain containing $p$ in its interior. If $K_{0} \cap \operatorname{bd} B \neq \emptyset$, then by compactness of bd $B$, there exists a $p_{0} \in K_{0} \cap S_{p q}$ such that $p_{0}$ is the only common point of $\mathrm{bd} B$ with the subarc $K$ of $K_{0}$ having $p$ and $p_{0}$ as its endpoints. If $K_{0} \cap \mathrm{bd} B=\emptyset$, then we put $K:=K_{0} \cap\left(S_{p q} \cup C_{p}\right)$. Take an $r_{B}>0$ such that $B\left(p, r_{B}\right) \subset$ int $B$ and $B\left(p, r_{B}\right) \cap S_{p q}$ is contained in the union of all trajectories having a common point with $K$, where $B\left(p, r_{B}\right)$ denotes the ball with centre $p$ and radius $r_{B}$. Fix a $T>0$ and an $r_{q}>0$. Without loss of generality we can assume that $B\left(q, r_{q}\right) \cap B\left(p, r_{B}\right)=\emptyset$.

Now we take an $r \in\left(0, r_{q}\right)$ for which there exists a $y \in$ $L_{0} \cap S_{p q}$ such that $\operatorname{dist}(q, y)>r$, where dist denotes the Euclidean metric on the plane. Then $\mathrm{bd} B(q, r) \cap L_{0} \cap S_{p q} \neq \emptyset$. By compactness of $\operatorname{bd} B(q, r)$, there exists a $q_{0} \in L_{0} \cap S_{p q}$ such that $q_{0}$ is the only common point of $\operatorname{bd} B(q, r)$ with the subarc $L$ of $L_{0}$ having $q$ and $q_{0}$ as its endpoints. Denote by $W$ the union of all trajectories having a common point with the $\operatorname{arc} L$. Since $q_{0} \in S_{p q}$, each trajectory contained in $W$ is a subset of the component of $\mathrm{cl} S_{q q_{0}} \backslash C_{p}$ which contains $C_{q}$ and $C_{q_{0}}$, where $S_{q q_{0}}$ denotes the strip between trajectories $C_{q}$ and $C_{q_{0}}$ of points $q$ and $q_{0}$.

By the assumption that $q \in J^{+}(p)$, there exist sequences $\left(p_{n}\right)_{n \in \mathbb{Z}_{+}}$and $\left(t_{n}\right)_{n \in \mathbb{Z}_{+}}$such that $p_{n} \rightarrow p, t_{n} \rightarrow+\infty$, $f^{t_{n}}\left(p_{n}\right) \rightarrow q$ as $n \rightarrow+\infty$. Thus there exists an $n_{0} \in \mathbb{Z}_{+}$ such that for all $n>n_{0}$ we have $t_{n}>T, p_{n} \in B\left(p, r_{B}\right)$ and $f^{t_{n}}\left(p_{n}\right) \in B(q, r) \cap W$. Then, for every $n>n_{0}$ there exists $\alpha_{n} \in \mathbb{R}$ such that $f^{t_{n}+\alpha_{n}}\left(p_{n}\right) \in L$. Moreover, by the definition of $r_{B}$, for every $n>n_{0}$ there exists $x_{n} \in K$ and $\beta_{n} \in \mathbb{R}$ such that $f^{\beta_{n}}\left(x_{n}\right)=p_{n}$. Thus $f^{t_{n}+\alpha_{n}+\beta_{n}}\left(x_{n}\right) \in L$ for $n>n_{0}$.

Fix any $n>n_{0}$ and take a positive integer $k_{n}$ such that $k_{n}>t_{n}+\alpha_{n}+\beta_{n}$ and $k_{n}>T$. Then $x_{n}$ and $f^{k_{n}}\left(x_{n}\right)$ belong to different components of $W \backslash L$, since $L$ is a section in $W$. By continuity of $f^{k_{n}}$ at $p$ there exists a $y_{n} \in f^{k_{n}}(K)$ such that $x_{n}$ and $y_{n}$ belong to the same component of $W \backslash L$, since any neighbourhood of $f^{k_{n}}(p)$ must contain a point from $f^{k_{n}}(K)$. Thus $f^{k_{n}}(K)$ has a common point $w_{n}$ with $L$. Then $w_{n} \in B(q, r)$ and hence $w_{n} \in B\left(q, r_{q}\right)$. Taking $z_{n}=f^{-k_{n}}\left(w_{n}\right)$ we have $z_{n} \in B$, since $K \subset B$. Consequently, for each $n>n_{0}$ we have $k_{n}>T$ and $f^{k_{n}}\left(z_{n}\right) \in B(q, r)$. Hence $k_{n} \rightarrow+\infty$ and $f^{k_{n}}\left(z_{n}\right) \rightarrow q$ as $n \rightarrow+\infty$, which implies that $q \in \omega_{f}(B)$. Consequently $q \in P^{+}(p)$.

Since an analogous reasoning can be applied to the set of strongly negatively irregular points and the first negative prolongational limit set, our considerations can be summarized in the following way.

Corollary 3. Let $f$ be a Brouwer homeomorphism which is embeddable in a flow $\left\{f^{t}: t \in \mathbb{R}\right\}$ and let $p \in \mathbb{R}^{2}$. Then $P^{+}(p)=J^{+}(p)$ and $P^{-}(p)=J^{-}(p)$, and consequently the set of all strongly irregular points of $f$ is equal to the first prolongational limit set of the flow $\left\{f^{t}: t \in \mathbb{R}\right\}$.

Corollary 4. Let $f$ be a Brouwer homeomorphism which is embeddable in a flow. Then, for each flow containing $f$, the first prolongational limit set is the same.

After a reparametrization of the flow $\left\{f^{t}: t \in \mathbb{R}\right\}$ containing $f$ each element $f^{t}$ of the flow, for $t \in \mathbb{R} \backslash\{0\}$ or $t>0$, respectively, can be treated as $f$.

Corollary 5. Let $f$ be a Brouwer homeomorphism which is embeddable in a flow $\left\{f^{t}: t \in \mathbb{R}\right\}$. Then the set of all strongly irregular points of $f^{t}$ is the same for all $t \in \mathbb{R} \backslash\{0\}$. Moreover, the set of all strongly positive irregular points of $f^{t}$ and the set of all strongly negative irregular points of $f^{t}$ are the same for all $t>0$.

\section{Flows of Brouwer Homeomorphisms}

In this section we describe the form of any flow of Brouwer homeomorphisms. To give a sufficient condition for the topological conjugacy of flows of Brouwer homeomorphisms one can use covers of the plane by maximal parallelizable regions. We will study the functions which express the relations between parallelizing homeomorphisms of such regions.

It is known that a simply connected region $U$ is parallelizable if and only if $J(U) \cap U=\emptyset$. Hence for every parallelizable region $U$ we have $J(U) \subset$ bd $U$. In the case where $U$ is a maximal parallelizable region (i.e., $U$ is not contained properly in any parallelizable region), the boundary of $U$ consists of strongly irregular points. It follows from the fact that for each maximal parallelizable region $U$ the equality $J(U)=$ bd $U$ holds. The proof of this fact can be found in [9]. For the convenience of the reader, we outline the essential ideas in that proof.

Let $U$ be a parallelizable region. Assume that there exists a point $p \in \operatorname{bd} U$ such that $p \notin J(U)$. Denote by $D_{1}$ the component of $\mathbb{R}^{2} \backslash C_{p}$ which has a common point with $U$ and by $D_{2}$ the other component of $\mathbb{R}^{2} \backslash C_{p}$. Let $V$ be a parallelizable region which contains $p$ and put $V_{1}:=V \cap D_{2}$. Let $U_{1}:=$ $U \cup C_{p} \cup V_{1}$. We show that $J(q) \cap U_{1}=\emptyset$ for each $q \in U_{1}$, which means that $U_{1}$ is a parallelizable region. To see this we consider three cases. First, let us consider the case where $q \epsilon$ $U$. Then $J(q) \subset \operatorname{cl} D_{1}$, since $q \in D_{1}$. Hence by parallelizability of $U$, we have $J(q) \cap U=\emptyset$ and by the assumption that $p \notin J(U)$, we get $J(q) \cap C_{p}=\emptyset$. Thus $J(q) \cap U_{1}=\emptyset$. Now, let $q \in V_{1}$. Then $J(q) \subset \operatorname{cl} D_{2}$. Hence $J(q) \cap U_{1}=\emptyset$, since by parallelizability of $V$ we have $J(q) \cap\left(C_{p} \cup V_{1}\right)=\emptyset$. Finally, let $q \in C_{p}$. Then, as in the previous case, $J(q) \cap\left(C_{p} \cup V_{1}\right)=\emptyset$, and by the assumption that $p \notin J(U)$, we get $J(q) \cap U=\emptyset$. Thus we proved that $J\left(U_{1}\right) \cap U_{1}=\emptyset$, which means that $U_{1}$ is parallelizable. Since $U$ is contained properly in $U_{1}$, we obtain that $U$ cannot be a maximal parallelizable region.

For any distinct trajectories $C_{p_{1}}, C_{p_{2}}$, and $C_{p_{3}}$ of $\left\{f^{t}: t \epsilon\right.$ $\mathbb{R}\}$ one of the following two possibilities must be satisfied: exactly one of the trajectories $C_{p_{1}}, C_{p_{2}}$, and $C_{p_{3}}$ is contained 
in the strip between the other two or each of the trajectories $C_{p_{1}}, C_{p_{2}}$, and $C_{p_{3}}$ is contained in the strip between the other two. In the first case if $C_{p_{j}}$ is the trajectory which lies in the strip between $C_{p_{i}}$ and $C_{p_{k}}$ we will write $C_{p_{i}}\left|C_{p_{j}}\right| C_{p_{k}}(i, j$, $k \in\{1,2,3\}$ and $i, j, k$ are different). In the second case we will write $\left|C_{p_{i}}, C_{p_{j}}, C_{p_{k}}\right|$ (cf. [10]).

Let $X$ be a nonempty set. Denote by $X^{<\omega}$ the set of all finite sequences of elements of $X$. A subset $T$ of $X^{<\omega}$ is called a tree on $X$ if it is closed under initial segments; that is, for all positive integers $m, n$ such that $n>m$ if $\left(x_{1}, \ldots, x_{m}, \ldots, x_{n}\right) \in$ $T$, then $\left(x_{1}, \ldots, x_{m}\right) \in T$. Let $\alpha=\left(x_{1}, \ldots, x_{n}\right) \in X^{<\omega}$. Then, for any $x \in X$ by $\alpha-x$ we denote the sequence $\left(x_{1}, \ldots, x_{n}, x\right)$. A node $\alpha=\left(x_{1}, \ldots, x_{n}\right) \in T$ of a tree $T$ is said to be terminal if there is no node of properly extending it; that is, there is no element $x \in X$ such that $\widehat{\alpha} x \in T$.

A tree $A^{+} \subset \mathbb{Z}_{+}^{<\omega}$ will be termed admissible if the following conditions hold:

(i) $A^{+}$contains the sequence 1 and no other one-element sequence;

(ii) if $\alpha^{-} k$ is in $A^{+}$and $k>1$, then so also is $\alpha^{-}(k-1)$.

A tree $A^{-} \subset \mathbb{Z}_{-}^{<\omega}$ will be termed admissible if the following conditions hold:

(iii) $A^{-}$contains the sequence -1 and no other oneelement sequence;

(iv) if $\alpha^{-} k$ is in $A^{-}$and $k<-1$, then so also is $\alpha^{-}(k+1)$.

The set $A:=A^{+} \cup A^{-}$will be said to be admissible class of finite sequences, where $A^{+}$and $A^{-}$are some admissible classes of finite sequences of positive and negative integers, respectively.

Now we recall results describing the flows of Brouwer homeomorphisms.

Theorem 6 (see [11]). Let $\left\{f^{t}: t \in \mathbb{R}\right\}$ be a flow of Brouwer homeomorphisms. Then there exists a family of trajectories $\left\{C_{\alpha}: \alpha \in A\right\}$ and a family of maximal parallelizable regions $\left\{U_{\alpha}: \alpha \in A\right\}$, where $A=A^{+} \cup A^{-}$is an admissible class of finite sequences, such that $U_{1}=U_{-1}, C_{1}=C_{-1}$, and

$$
\begin{aligned}
& C_{\alpha} \subset U_{\alpha} \text { for } \alpha \in A \text {, } \\
& \bigcup_{\alpha \in A} U_{\alpha}=\mathbb{R}^{2} \\
& U_{\alpha} \cap U_{\alpha^{-i}} \neq \emptyset \text { for } \alpha \widetilde{i} \in A \text {, } \\
& C_{\alpha \sim i} \subset b d U_{\alpha} \text { for } \alpha \widehat{i} \in A \text {, } \\
& \left|C_{\alpha}, C_{\alpha i_{1}}, C_{\alpha i_{2}}\right| \quad \text { for } \alpha \widehat{i_{1}}, \widehat{\alpha i_{2}} \in A, i_{1} \neq i_{2} \text {, }
\end{aligned}
$$

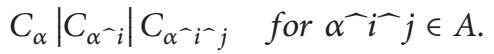

Proposition 7 (see [11]). Let $\left\{f^{t}: t \in \mathbb{R}\right\}$ be a flow of Brouwer homeomorphisms. Then there exists a family of the parallelizing homeomorphisms $\left\{\varphi_{\alpha}: \alpha \in A^{+}\right\}$, where $\varphi_{\alpha}: U_{\alpha} \rightarrow \mathbb{R}^{2}, U_{\alpha}$ are those occurring in Theorem 6 , and for each $\alpha^{-} i \in A^{+}$

$$
\begin{gathered}
\varphi_{\alpha \sim i}\left(U_{\alpha} \cap U_{\alpha^{-} i}\right)=\mathbb{R} \times\left(c_{\alpha^{-} i}, 0\right), \\
\varphi_{\alpha}\left(U_{\alpha} \cap U_{\alpha^{-} i}\right)=\mathbb{R} \times\left(c_{\alpha}, d_{\alpha}\right),
\end{gathered}
$$

where $c_{\alpha} \in \mathbb{R} \cup\{-\infty\}, d_{\alpha} \in \mathbb{R} \cup\{+\infty\}$, and $c_{\alpha^{-} i} \in[-\infty, 0)$ are some constants such that $c_{\alpha}<d_{\alpha}$ and at least one of the constants $c_{\alpha}, d_{\alpha}$ is finite. Moreover, there exists a continuous function $\mu_{\alpha \wedge_{i}}:\left(c_{\alpha}, d_{\alpha}\right) \rightarrow \mathbb{R}$ and a homeomorphism $\nu_{\alpha{ }^{-i}}:$ $\left(c_{\alpha}, d_{\alpha}\right) \rightarrow\left(c_{\alpha^{-} i}, 0\right)$ such that the homeomorphism

$$
h_{\alpha^{-} i}: \mathbb{R} \times\left(c_{\alpha}, d_{\alpha}\right) \longrightarrow \mathbb{R} \times\left(c_{\alpha^{-} i}, 0\right)
$$

given by the relation $h_{\alpha^{-} i}:=\varphi_{\alpha^{-} i} \circ\left(\varphi_{\left.\alpha\right|_{U_{\alpha} \cap U_{\alpha^{\wedge} i}}}\right)^{-1}$ has the form

$$
h_{\alpha^{-i} i}(t, s)=\left(\mu_{\alpha^{-} i}(s)+t, \nu_{\alpha^{-\imath} i}(s)\right), \quad t \in \mathbb{R}, s \in\left(c_{\alpha}, d_{\alpha}\right) \text {. }
$$

The above proposition is formulated for $\alpha \in A^{+}$, but the analogous result holds for $\alpha \in A^{-}$. The admissible class of finite sequences occurring in Theorem 6 is not unique for a given flow, so we can usually choose a convenient $A$ when solving a problem of topological conjugacy.

The homeomorphisms $v_{\alpha^{-} i}$ occurring in Proposition 7 can be either increasing or decreasing. For each $\alpha \widehat{-i} \in A$ denote by $C_{\alpha^{-} i}^{\alpha}$ the unique trajectory contained in $U_{\alpha} \cap$ $J\left(C_{\alpha^{-} i}\right)$ (the uniqueness has been proven in [8]). From the construction of the families $\left\{C_{\alpha}: \alpha \in A\right\}$ and $\left\{U_{\alpha}\right.$ : $\alpha \in A\}$ occurring in Theorem 6 we obtain that, in case $C_{\alpha}\left|C_{\alpha^{-} i}^{\alpha}\right| C_{\alpha^{-} i}$ or $C_{\alpha}=C_{\alpha^{-} i}^{\alpha}$, the homeomorphism $\nu_{\alpha^{-} i}$ is decreasing and $c_{\alpha}>0$ or $c_{\alpha}=0$, respectively. However, in case $\left|C_{\alpha}, C_{\alpha^{-} i}^{\alpha}, C_{\alpha^{-} i}\right|$, the homeomorphism $\nu_{\alpha^{-i}}$ is increasing and $d_{\alpha}>0$ (see [11]).

The continuous functions $\mu_{\alpha-i}$ describe the time needed for the flow $\left\{f^{t}: t \in \mathbb{R}\right\}$ to move from the point with coordinates $\left(0, v_{\alpha^{-} i}(s)\right)$ in the chart $\varphi_{\alpha^{-} i}$ until it reaches the point with coordinates $(0, s)$ in the chart $\varphi_{\alpha}$. In other words, $\mu_{\alpha_{-i}}$ describe the time needed for the flow to move from a point from the section $K_{\varphi_{\alpha-i}}$ in $U_{\alpha^{-} i}$ to a point from the section $K_{\varphi_{\alpha}}$ in $U_{\alpha}$.

Proposition 8. The functions $\mu_{\alpha^{-i}}$ occurring in Proposition 7 satisfy the condition

$$
\lim _{s \rightarrow c_{\alpha}} \mu_{\alpha^{-} i}(s)= \begin{cases}-\infty & \text { if } C_{\alpha^{-i}} \subset J^{+}\left(C_{\alpha^{-} i}^{\alpha}\right), \\ +\infty & \text { if } C_{\alpha^{-} i} \subset J^{-}\left(C_{\alpha^{-} i}\right)\end{cases}
$$

in the case where $C_{\alpha}\left|C_{\alpha^{-} i}^{\alpha}\right| C_{\alpha^{-i} i}$ or $C_{\alpha}=C_{\alpha^{-} i}^{\alpha}$ or the condition

$$
\lim _{s \rightarrow d_{\alpha}} \mu_{\alpha^{-i}}(s)= \begin{cases}-\infty & \text { if } C_{\alpha^{-} i} \subset J^{+}\left(C_{\alpha^{-i}}^{\alpha}\right), \\ +\infty & \text { if } C_{\alpha^{-} i} \subset J^{-}\left(C_{\alpha^{-i} i}\right)\end{cases}
$$

in the case where $\left|C_{\alpha^{\prime}}, C_{\alpha^{-} i}^{\alpha}, C_{\alpha^{-} i}\right|$.

Proof. Let us consider the case where $C_{\alpha}\left|C_{\alpha^{-} i}^{\alpha}\right| C_{\alpha^{-} i}$ or $C_{\alpha}=$ $C_{\alpha^{-} i}^{\alpha}$ and assume that $C_{\alpha^{-} i} \subset J^{+}\left(C_{\alpha^{-} i}^{\alpha}\right)$. The other cases are similar. Denote by $p$ and $q$ the points for which $\varphi_{\alpha}(p)=\left(0, c_{\alpha}\right)$ and $\varphi_{\alpha^{-} i}(q)=(0,0)$; that is, $p \in K_{\varphi_{\alpha}} \cap C_{\alpha^{-} i}^{\alpha}$ and $q \in K_{\varphi_{\alpha^{-} i}} \cap$ $C_{\alpha \wedge i}$. Then $q \in J^{+}(p)$. Thus there exist sequences $\left(p_{n}\right)_{n \in \mathbb{Z}_{+}}$and $\left(t_{n}\right)_{n \in \mathbb{Z}_{+}}$such that $p_{n} \rightarrow p, t_{n} \rightarrow+\infty$, and $f^{t_{n}}\left(p_{n}\right) \rightarrow q$ as $n \rightarrow+\infty$. This means that there exist sequences $\left(u_{n}\right)_{n \in \mathbb{Z}_{+}}$, $\left(s_{n}\right)_{n \in \mathbb{Z}_{+}}$such that $u_{n} \rightarrow 0, s_{n} \rightarrow c_{\alpha}$, where $\varphi_{\alpha}\left(p_{n}\right)=\left(u_{n}, s_{n}\right)$. Hence $\varphi_{\alpha}\left(f^{t_{n}}\left(p_{n}\right)\right)=\varphi_{\alpha}\left(p_{n}\right)+\left(t_{n}, 0\right)=\left(t_{n}+u_{n}, s_{n}\right)$ and by (11)

$$
h_{\alpha^{-} i}\left(t_{n}+u_{n}, s_{n}\right)=\left(\mu_{\alpha^{-} i}\left(s_{n}\right)+t_{n}+u_{n}, v_{\alpha^{-} i}\left(s_{n}\right)\right) .
$$


Thus $\mu_{\alpha{ }^{-} i}\left(s_{n}\right)+t_{n}+u_{n} \rightarrow 0$ as $n \rightarrow+\infty$, since $f^{t_{n}}\left(p_{n}\right) \rightarrow q$ as $n \rightarrow+\infty$. Hence $\mu_{\alpha-i}\left(s_{n}\right) \rightarrow-\infty$, since $t_{n} \rightarrow+\infty$ and $u_{n} \rightarrow 0$. Consequently, $\lim \inf _{s \rightarrow c_{\alpha}} \mu_{\alpha \wedge i}(s)=-\infty$.

Suppose, on the contrary, that there exists a sequence $\left(s_{n}\right)_{n \in \mathbb{Z}_{+}}$such that $s_{n} \rightarrow c_{\alpha}$ and $\mu_{\alpha^{-} i}\left(s_{n}\right) \rightarrow c$ for some $c \in \mathbb{R}$. Consider the sequence $\left(p_{n}\right)_{n \in \mathbb{Z}_{+}}$such that $\varphi_{\alpha}\left(p_{n}\right)=\left(0, s_{n}\right)$. Then each element of the sequence $\left(p_{n}\right)_{n \in \mathbb{Z}_{+}}$belongs to $K_{\varphi_{\alpha}}$. Moreover, the sequence $\left(p_{n}\right)_{n \in \mathbb{Z}_{+}}$tends to the point $p$ such that $\varphi_{\alpha}(p)=\left(0, c_{\alpha}\right)$. Hence $p \in K_{\varphi_{\alpha}} \cap C_{\alpha^{-} i}^{\alpha}$. On the other hand, by (11)

$$
\varphi_{\alpha^{\wedge} i}\left(p_{n}\right)=h_{\alpha^{-i}}\left(0, s_{n}\right)=\left(\mu_{\alpha^{-i}}\left(s_{n}\right), \nu_{\alpha^{-i}}\left(s_{n}\right)\right) .
$$

Hence $\lim _{n \rightarrow+\infty} \varphi_{\alpha^{-} i}\left(p_{n}\right)=(c, 0)$. Consequently $\lim _{n \rightarrow+\infty} p_{n}$ $=\widetilde{q}$, where $\widetilde{q}$ is a point such that $\varphi_{\alpha^{-} i}(\widetilde{q})=(c, 0)$; that is, $\widetilde{q} \in$ $C_{\alpha^{\wedge} i}$. But this is impossible, since $C_{\alpha^{\wedge} i} \cap C_{\alpha^{\wedge} i}^{\alpha}=\emptyset$.

By the fact that $\nu_{\alpha^{-} i}:\left(c_{\alpha}, d_{\alpha}\right) \rightarrow\left(c_{\alpha^{-} i}, 0\right)$ is a homeomorphism, the function $\widetilde{h}_{\alpha^{-} i}: \mathbb{R} \times\left(c_{\alpha^{-} i}, 0\right) \rightarrow \mathbb{R} \times$ $\left(c_{\alpha \sim i}, 0\right)$ defined by

$$
\widetilde{h}_{\alpha^{-} i}(t, s):=\left(\left(\mu_{\alpha^{-} i} \circ \nu_{\alpha^{-} i}^{-1}\right)(v)+t, v\right), \quad t \in \mathbb{R}, v \in\left(c_{\alpha^{-} i}, 0\right)
$$

is continuous. Moreover, putting $s:=\nu_{\alpha^{-} i}^{-1}(v)$ in Proposition 8 we obtain the following result.

Corollary 9. The functions $\eta_{\alpha^{-} i}:\left(c_{\alpha^{-} i}, 0\right) \rightarrow \mathbb{R}$ given by

$$
\eta_{\alpha^{-} i}:=\mu_{\alpha^{-} i} \circ \nu_{\alpha^{-} i}^{-1}
$$

where $\mu_{\alpha^{-i}}$ and $\nu_{\alpha^{-i}}$ are those occurring in Proposition 7, satisfy the condition

$$
\lim _{v \rightarrow 0} \eta_{\alpha^{-} i}(v)= \begin{cases}-\infty & \text { if } C_{\alpha^{-} i} \subset J^{+}\left(C_{\alpha^{-} i}^{\alpha}\right), \\ +\infty & \text { if } C_{\alpha^{-} i} \subset J^{-}\left(C_{\alpha^{-} i}\right) .\end{cases}
$$

\section{Topological Conjugacy of Generalized Reeb Flows}

In this section we consider the problem of topological conjugacy of a class of flows of Brouwer homeomorphisms. To prove our result we use the form of such flows.

We say that flows $\left\{f^{t}: t \in \mathbb{R}\right\}$ and $\left\{g^{t}: t \in \mathbb{R}\right\}$, where $f^{t}, g^{t}: \mathbb{R}^{2} \rightarrow \mathbb{R}^{2}$, are topologically conjugate if there exists a homeomorphism $\Phi$ of the plane onto itself such that

$$
g^{t}=\Phi^{-1} \circ f^{t} \circ \Phi, \quad t \in \mathbb{R} .
$$

In [12] a lemma can be found which says that the set of strongly irregular points (called the set of singular pairs there) is invariant with respect to topological conjugacy of flows. Thus, by Corollary 3, we have the following result.

Proposition 10. Let $\left\{f^{t}: t \in \mathbb{R}\right\}$ and $\left\{g^{t}: t \in \mathbb{R}\right\}$ be topologically conjugate flows of Brouwer homeomorphisms and let $\Phi: \mathbb{R}^{2} \rightarrow \mathbb{R}^{2}$ be a homeomorphism which conjugates the flows. Then $\Phi\left(J_{\left\{f^{t}\right\}}\left(\mathbb{R}^{2}\right)\right)=J_{\left\{g^{t}\right\}}\left(\mathbb{R}^{2}\right)$, where $J_{\left\{f^{t}\right\}}\left(\mathbb{R}^{2}\right)$ and $J_{\left\{g^{t}\right\}}\left(\mathbb{R}^{2}\right)$ denote the first prolongational limit set of $\left\{f^{t}: t \in \mathbb{R}\right\}$ and $\left\{g^{t}: t \in \mathbb{R}\right\}$, respectively.
Put

$$
\begin{gathered}
P_{0}:=\left\{(x, y) \in \mathbb{R}^{2}: x>0, y>0\right\}, \\
P_{1}:=\left\{(x, y) \in \mathbb{R}^{2}: x<0, y>0\right\}, \\
P_{2}:=\left\{(x, y) \in \mathbb{R}^{2}: x>0, y<0\right\}, \\
L_{x}:=\left\{(x, 0) \in \mathbb{R}^{2}: x>0\right\}, \\
L_{y}:=\left\{(0, y) \in \mathbb{R}^{2}: y>0\right\}
\end{gathered}
$$

and $U:=P_{0} \cup P_{1} \cup P_{2} \cup L_{x} \cup L_{y}$. Consider the flow $\left\{g^{t}: t \in \mathbb{R}\right\}$, where for each $t \in \mathbb{R}$ the homeomorphism $g^{t}: U \rightarrow U$ is defined by

$$
g^{t}(x, y):= \begin{cases}\left(2^{t} x, 2^{-t} y\right) & \text { if }(x, y) \in P_{0} \cup L_{x} \cup L_{y} \\ \left(x, 2^{-t} y\right) & \text { if }(x, y) \in P_{1} \\ \left(2^{t} x, y\right) & \text { if }(x, y) \in P_{2}\end{cases}
$$

Then $J^{+}(U)=L_{x}$ and $J^{-}(U)=L_{y}$.

Put $A^{+}:=\{1,(1,1)\}, U_{1}:=P_{1} \cup L_{y} \cup P_{0}, U_{(1,1)}:=P_{0} \cup$ $L_{x} \cup P_{2}, C_{1}:=L_{y}, C_{(1,1)}:=L_{x}$ and $A^{-}:=\{-1\}, U_{-1}:=U_{1}$, $C_{-1}:=C_{1}$. Then $C_{(1,1)}^{1}:=C_{1}$ and $C_{(1,1)} \subset J^{+}\left(C_{1}\right)$. Let

$$
K_{\varphi_{1}}:=\{(s, 1): s \in \mathbb{R}\}, \quad K_{\varphi_{(1,1)}}:=\{(1, s): s \in \mathbb{R}\} .
$$

Note that the trajectories of $\left\{g^{t}: t \in \mathbb{R}\right\}$ contained in $P_{0}$ are given by the equation $x y=s$ for $s \in(0,+\infty)$. Hence

$$
\mu_{(1,1)}:(0,+\infty) \longrightarrow \mathbb{R}, \quad \mu_{(1,1)}(s)=\log _{2} s,
$$

since $g^{\log _{2} s}(1, s)=\left(2^{\log _{2} s}, 2^{-\log _{2} s} s\right)=(s, 1)$. Moreover,

$$
\nu_{(1,1)}:(0,+\infty) \longrightarrow(-\infty, 0), \quad \nu_{(1,1)}(s)=-s
$$

For each flow $\left\{f^{t}: t \in \mathbb{R}\right\}$, where $f^{t}: U \rightarrow U$ for $t \in \mathbb{R}$, having the same trajectories (including the orientation) as the flow $\left\{g^{t}: t \in \mathbb{R}\right\}$ given by (21), one can consider the function $\mu_{\left\{f^{t}\right\},(1,1)}:(0,+\infty) \rightarrow \mathbb{R}$ occurring in Proposition 7 which describes the time needed to move from each point $p \in P_{0} \cap$ $K_{\varphi_{(1,1)}}$ to the point of $K_{\varphi_{1}}$ belonging to the trajectory of $p$, that is, from the point of the form $(1, s)$ to the point of the form $(s, 1)$ for some $s \in(0,+\infty)$. Then by Proposition 8

$$
\lim _{s \rightarrow 0} \mu_{\left\{f^{t}\right\},(1,1)}(s)=-\infty \text {. }
$$

Consider a constant $\sigma\left(\mu_{\left\{f^{t}\right\},(1,1)}\right) \in[0,+\infty]$ defined by

$$
\sigma\left(\mu_{\left\{f^{t}\right\},(1,1)}\right):=\limsup _{v \rightarrow 0} \mu_{\left\{f^{t}\right\},(1,1)}^{*}(v)
$$

where $\mu_{\left\{f^{t}\right\},(1,1)}^{*}:(0,1] \rightarrow[0,+\infty)$ is given by

$$
\mu_{\left\{f^{t}\right\},(1,1)}^{*}(v):=\mu_{\left\{f^{t}\right\},(1,1)}(v)-\min \left\{\mu_{\left\{f^{t}\right\},(1,1)}(s): s \in[v, 1]\right\}
$$

(cf. $[13,14])$. Then the flow $\left\{f^{t}: t \in \mathbb{R}\right\}$ is topologically conjugate to the flow $\left\{g^{t}: t \in \mathbb{R}\right\}$ given by (21) if and only if 
$\sigma\left(\mu_{\left\{f^{t}\right\},(1,1)}\right)=0$ (see [13]). In particular, this condition holds in the case where $\mu_{\left\{f^{t}\right\},(1,1)}$ is increasing.

Now we introduce a class of flows of Brouwer homeomorphisms. Put $\alpha_{1}:=1$ and $\alpha_{n+1}:=\alpha_{n} \widehat{-1}$ for $n \in \mathbb{Z}_{+}$. For any positive integer $k$ we define $A_{k}:=\left\{\alpha_{n}: 1 \leq n \leq k\right\}$ and $A_{+\infty}:=\left\{\alpha_{n}: n \in \mathbb{Z}_{+}\right\}$. Similarly, put $\alpha_{-1}:=-1$, $\alpha_{n-1}:=\alpha_{n}{ }^{-}-1$ for $n \in \mathbb{Z}_{-}$and for any negative integer $k$ let $A_{k}:=\left\{\alpha_{n}: k \leq n \leq-1\right\}$ and $A_{-\infty}:=\left\{\alpha_{n}: n \in \mathbb{Z}_{-}\right\}$.

Consider a flow $\left\{h^{t}: t \in \mathbb{R}\right\}$ of Brouwer homeomorphisms $h^{t}: \mathbb{R}^{2} \rightarrow \mathbb{R}^{2}$ such that $A=A^{+} \cup A^{-}$can be given in one of the following forms:

(a) $A^{-}=\{-1\}$ and $A^{+}=A_{k}$ for some $k \in \mathbb{Z}_{+}$,

(b) $A^{-}=\{-1\}$ and $A^{+}=A_{+\infty}$,

(c) $A^{-}=A_{-\infty}$ and $A^{+}=A_{+\infty}$.

We assume that $U_{\alpha_{n}} \cap J\left(\mathbb{R}^{2}\right)=C_{\alpha_{n}}$ for each $\alpha_{n} \in A$, where $\left\{U_{\alpha_{n}}: \alpha_{n} \in A\right\}$ and $\left\{C_{\alpha_{n}}: \alpha_{n} \in A\right\}$ are whose occurring in Theorem 6. Then $C_{\alpha_{n+1}}^{\alpha_{n}}=C_{\alpha_{n}}$, since $C_{\alpha_{n+1}}^{\alpha_{n}} \subset U_{\alpha_{n}} \cap J\left(\mathbb{R}^{2}\right)$ for every $\alpha_{n+1} \in A^{+}$. Similarly, $C_{\alpha_{n-1}}^{\alpha_{n}}=C_{\alpha_{n}}$ for every $\alpha_{n-1} \in A^{-}$.

Fix an $\alpha_{n+1} \in A^{+}$. Denote by $V_{\alpha_{n+1}}$ the strip between $C_{\alpha_{n}}$ and $C_{\alpha_{n+1}}$. Then $V_{\alpha_{n+1}} \subset U_{\alpha_{n}}$ and $\left|C, C_{\alpha_{n}}, C_{\alpha_{n+1}}\right|$ for every trajectory $C \subset V_{\alpha_{n+1}}$ (see [8]). In particular, if $C_{\alpha_{n}}$ is equal to the vertical line $\{(n-1, y): y \in \mathbb{R}\}$ for each $\alpha_{n} \in A^{+}$, then $V_{\alpha_{n+1}}$ is a vertical strip for each $\alpha_{n+1} \in A^{+}$. In a similar way we define the strip $V_{\alpha_{n-1}}$ for $\alpha_{n-1} \in A^{-}$.

Let us assume that for each $\alpha_{n} \in A \backslash\{1,-1\}$ there exists a homeomorphism $\psi_{\alpha_{n}}: \operatorname{cl} V_{\alpha_{n}} \rightarrow P_{0} \cup L_{x} \cup L_{y}$ such that

$$
h^{t}=\psi_{\alpha_{n}}^{-1} \circ g^{t} \circ \psi_{\alpha_{n}}, \quad t \in \mathbb{R},
$$

where $\left\{g^{t}: t \in \mathbb{R}\right\}$ is given by (21). If $C_{\alpha_{n}} \subset J^{+}\left(C_{\alpha_{n-1}}\right)$, then $\psi_{\alpha_{n}}\left(C_{\alpha_{n-1}}\right)=L_{y}$ and $\psi_{\alpha_{n}}\left(C_{\alpha_{n}}\right)=L_{x}$. In case $C_{\alpha_{n}} \subset J^{-}\left(C_{\alpha_{n-1}}\right)$ we have $\psi_{\alpha_{n}}\left(C_{\alpha_{n-1}}\right)=L_{x}$ and $\psi_{\alpha_{n}}\left(C_{\alpha_{n}}\right)=L_{y}$. The flow $\left\{h^{t}: t \epsilon\right.$ $\mathbb{R}\}$ described above will be called a standard generalized Reeb flow.

A standard generalized Reeb flow can have either a finite number of maximal parallelizable regions or an infinite number of such regions. The first case holds if the set of indices $A$ of the flow is of the form (a). However, the second case holds if this set is of the form (b) or (c). The trajectories of a standard generalized Reeb flow with an infinite number of maximal parallelizable regions are shown in Figures 1 and 2 for the set $A$ of the forms (b) and (c), respectively.

Consider a flow $\left\{f^{t}: t \in \mathbb{R}\right\}$ of Brouwer homeomorphisms which has the same trajectories as a standard generalized Reeb flow. For $\alpha_{n} \in A \backslash\{1,-1\}$ and $s \in(0,+\infty)$ denote by $C_{s}^{\alpha_{n}}$ the image of the trajectory $\left\{(x, y) \in P_{0}: x y=s\right\}$ of $\left\{g^{t}: t \in \mathbb{R}\right\}$ under $\psi_{\alpha_{n}}^{-1}$. For each $\alpha_{n} \in A \backslash\{1,-1\}$ consider the function $\mu_{\left\{f^{t}\right\}, \alpha_{n}}:(0,+\infty) \rightarrow \mathbb{R}$ taking as $\mu_{\left\{f^{t}\right\}, \alpha_{n}}(s)$ the time needed to move from the unique point of the set $C_{s}^{\alpha_{n}} \cap \psi_{\alpha_{n}}^{-1}\left(K_{\varphi_{(1,1)}}\right)$ to the unique point of $C_{s}^{\alpha_{n}} \cap \psi_{\alpha_{n}}^{-1}\left(K_{\varphi_{1}}\right)$. Define $\mu_{\left\{f^{t}\right\}, \alpha_{n}}^{*}:(0,1] \rightarrow[0,+\infty)$ by

$$
\mu_{\left\{f^{t}\right\}, \alpha_{n}}^{*}(v):=\mu_{\left\{f^{t}\right\}, \alpha_{n}}(v)-\min \left\{\mu_{\left\{f^{t}\right\}, \alpha_{n}}(s): s \in[v, 1]\right\}
$$

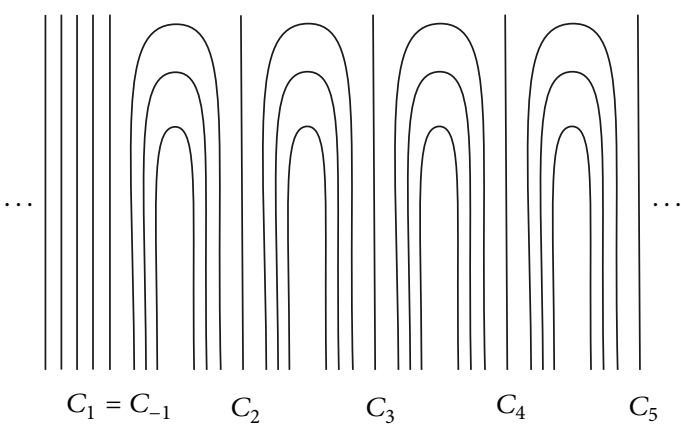

Figure 1: A generalized Reeb flow with $A^{-}=\{-1\}$ and $A^{+}=A_{+\infty}$.

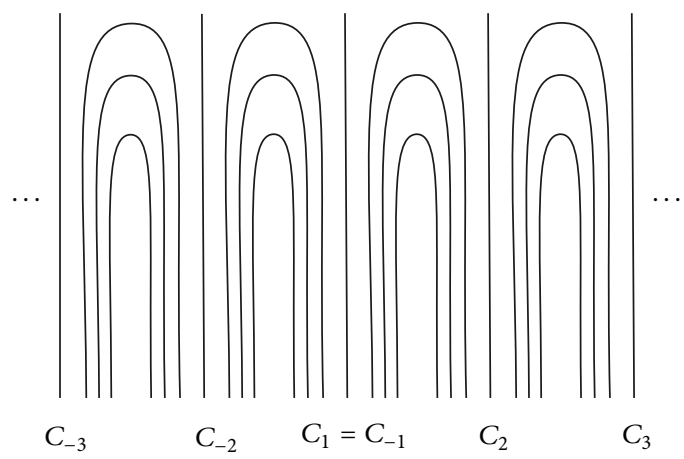

Figure 2: A generalized Reeb flow with $A^{-}=A_{-\infty}$ and $A^{+}=A_{+\infty}$.

in case $C_{\alpha_{n+1}} \subset J^{+}\left(C_{\alpha_{n}}\right)$, and by

$$
\mu_{\left\{f^{t}\right\}, \alpha_{n}}^{*}(v):=\max \left\{\mu_{\left\{f^{t}\right\}, \alpha_{n}}(s): s \in[v, 1]\right\}-\mu_{\left\{f^{t}\right\}, \alpha_{n}}(v)
$$

in case $C_{\alpha_{n+1}} \subset J^{-}\left(C_{\alpha_{n}}\right)$. Put

$$
\sigma\left(\mu_{\left\{f^{t}\right\}, \alpha_{n}}\right):=\limsup _{v \rightarrow 0} \mu_{\left\{f^{t}\right\}, \alpha_{n}}^{*}(v) .
$$

Now we can prove the following conjugacy result.

Theorem 11. Let $\left\{h^{t}: t \in \mathbb{R}\right\}$ be a standard generalized Reeb flow. Let $A$ be an admissible class of finite sequences satisfying one of the conditions (a)-(c). Assume that $\left\{f^{t}: t \in \mathbb{R}\right\}$ is a flow of Brouwer homeomorphisms having the same trajectories including orientation as $\left\{h^{t}: t \in \mathbb{R}\right\}$. If $\sigma\left(\mu_{\left\{f^{t}\right\}, \alpha_{n}}\right)=0$ for all $\alpha_{n} \in A \backslash\{1,-1\}$, then the flows $\left\{f^{t}: t \in \mathbb{R}\right\}$ and $\left\{h^{t}: t \in \mathbb{R}\right\}$ are topologically conjugate.

Proof. Assume that one of the conditions (a) and (b) holds. First, let us note that there exists a topological conjugacy $\Phi_{1}$ : $U_{1} \rightarrow U_{1}$ of flows $\left\{\left.f^{t}\right|_{U_{1}}: t \in \mathbb{R}\right\}$ and $\left\{\left.h^{t}\right|_{U_{1}}: t \in \mathbb{R}\right\}$, since $U_{1}$ is a parallelizable region of each of these flows. More precisely, if $\varphi_{\left\{f^{t}\right\}, 1}: U_{1} \rightarrow \mathbb{R}^{2}$ and $\varphi_{\left\{h^{t}\right\}, 1}: U_{1} \rightarrow \mathbb{R}^{2}$ are parallelizing homeomorphisms for $\left\{\left.f^{t}\right|_{U_{1}}: t \in \mathbb{R}\right\}$ and $\left\{\left.h^{t}\right|_{U_{1}}: t \in \mathbb{R}\right\}$, respectively, then for every $p \in U_{1}$ we put $\Phi_{1}(p):=\left(\varphi_{\left\{h^{t}\right\}, 1}^{-1}\right.$ 。 $\left.\varphi_{\left\{f^{t}\right\}, 1}\right)(p)$.

Fix an $\alpha_{n+1} \in A^{+}$. Assume that we have defined a homeomorphism $\Phi_{n}$ which conjugates $\left\{f^{t}: t \in \mathbb{R}\right\}$ and $\left\{h^{t}: t \in \mathbb{R}\right\}$ on the set $\bigcup_{i=1}^{n} U_{\alpha_{i}}$. Define $F^{t}: P_{0} \cup L_{x} \cup L_{y} \rightarrow$ 
$P_{0} \cup L_{x} \cup L_{y}$ by $F^{t}:=\psi_{\alpha_{n+1}} \circ f^{t} \circ \psi_{\alpha_{n+1}}^{-1}$ for $t \in \mathbb{R}$, where $\psi_{\alpha_{n+1}}$ satisfies (28). Then $\sigma\left(\mu_{\left\{F^{t}\right\},(1,1)}\right) \stackrel{\alpha_{n+1}}{=} \sigma\left(\mu_{\left\{f^{t}\right\}, \alpha_{n}}\right)$. Hence $\sigma\left(\mu_{\left\{F^{t}\right\},(1,1)}\right)=0$, since by the assumption $\sigma\left(\mu_{\left\{f^{t}\right\}, \alpha_{n}}\right)=0$. Thus $\left\{F^{t}: t \in \mathbb{R}\right\}$ and $\left\{\left.g^{t}\right|_{P_{0} \cup L_{x} \cup L_{y}}: t \in \mathbb{R}\right\}$ are topologically conjugate. Consequently $\left\{\left.f^{t}\right|_{W_{\alpha_{n+1}}}: t \in \mathbb{R}\right\}$ and $\left\{\left.h^{t}\right|_{W_{\alpha_{n+1}}}\right.$ : $t \in \mathbb{R}\}$ are topologically conjugate, where $W_{\alpha_{n+1}}:=\operatorname{cl} V_{\alpha_{n+1}}$. Denote by $\phi_{\alpha_{n+1}}$ the homeomorphism which conjugates these flows.

Fix any $p_{0} \in C_{\alpha_{n}}$ and put $q_{1}:=\Phi_{n}\left(p_{0}\right), q_{2}:=\phi_{\alpha_{n+1}}\left(p_{0}\right)$. Take $t_{0} \in \mathbb{R}$ such that $h^{t_{0}}\left(q_{2}\right)=q_{1}$ and define $\Phi_{\alpha_{n+1}}:=$ $\left.h^{t_{0}}\right|_{W_{\alpha_{n+1}}} \circ \phi_{\alpha_{n+1}}$. Then $\Phi_{\alpha_{n+1}}$ conjugates the flows $\left\{\left.f^{t}\right|_{W_{\alpha_{n+1}}}: t \in\right.$ $\mathbb{R}\}$ and $\left\{\left.h^{t}\right|_{W_{\alpha_{n+1}}}: t \in \mathbb{R}\right\}$, since

$$
\begin{aligned}
& \left.\Phi_{\alpha_{n+1}} \circ f^{t}\right|_{W_{\alpha_{n+1}}} \\
& =\left.\left.h^{t_{0}}\right|_{W_{\alpha_{n+1}}} \circ \phi_{\alpha_{n+1}} \circ f^{t}\right|_{W_{\alpha_{n+1}}} \\
& =\left.\left.h^{t_{0}}\right|_{W_{\alpha_{n+1}}} \circ h^{t}\right|_{W_{\alpha_{n+1}}} \circ \phi_{\alpha_{n+1}} \\
& =\left.\left.h^{t}\right|_{W_{\alpha_{n+1}}} \circ h^{t_{0}}\right|_{W_{\alpha_{n+1}}} \circ \phi_{\alpha_{n+1}}=\left.h^{t}\right|_{W_{\alpha_{n+1}}} \circ \Phi_{\alpha_{n+1}} .
\end{aligned}
$$

Moreover $\Phi_{\alpha_{n+1}}\left(p_{0}\right)=q_{1}$, since

$$
\Phi_{\alpha_{n+1}}\left(p_{0}\right)=h^{t_{0}}\left(\phi_{\alpha_{n+1}}\left(p_{0}\right)\right)=h^{t_{0}}\left(q_{2}\right)=q_{1} .
$$

Hence $\left.\Phi_{\alpha_{n+1}}\right|_{C_{\alpha_{n}}}=\left.\Phi_{n}\right|_{C_{\alpha_{n}}}$. Thus we can define $\Phi_{n+1}$ by

$$
\Phi_{n+1}(p):= \begin{cases}\Phi_{n}(p), & p \in \bigcup_{i=1}^{n} U_{\alpha_{i}} \backslash V_{\alpha_{n+1}}, \\ \Phi_{\alpha_{n+1}}(p), & p \in V_{\alpha_{n+1}} .\end{cases}
$$

Then $\Phi_{n+1}$ conjugates $\left\{f^{t}: t \in \mathbb{R}\right\}$ and $\left\{h^{t}: t \in \mathbb{R}\right\}$ on the set $\bigcup_{i=1}^{n} U_{\alpha_{i}} \cup C_{\alpha_{n+1}}$. Since $\left\{f^{t}: t \in \mathbb{R}\right\}$ and $\left\{h^{t}: t \in\right.$ $\mathbb{R}$ \} are parallelizable on $U_{\alpha_{n+1}}$ we can extend the topological conjugacy $\Phi_{n+1}$ on the component of $U_{\alpha_{n+1}} \backslash C_{\alpha_{n+1}}$ which do not contain $C_{\alpha_{n}}$ (see [12]). Such an extension is really needed in case of (a) to obtain the conjugacy on the whole plane. In case of (c), for any $\alpha_{n-1} \in A^{-}$we extend $\Phi_{n}$ from $\bigcup_{i=-1}^{n} U_{\alpha_{i}}$ to $\Phi_{n-1}$ defined on $\bigcup_{i=-1}^{n} U_{\alpha_{i}} \cup C_{\alpha_{n-1}}$ in a similar way.

\section{Conflict of Interests}

The author declares that there is no conflict of interests regarding the publication of this paper.

\section{References}

[1] H. Nakayama, "Limit sets and square roots of homeomorphisms," Hiroshima Mathematical Journal, vol. 26, no. 2, pp. 405-413, 1996.

[2] L. E. J. Brouwer, "Beweis des ebenen translationssatzes," Mathematische Annalen, vol. 72, no. 1, pp. 37-54, 1912.

[3] T. Homma and H. Terasaka, "On the structure of the plane translation of Brouwer," Osaka Journal of Mathematics, vol. 5, pp. 233-266, 1953.

[4] H. Nakayama, "On dimensions of non-Hausdorff sets for plane homeomorphisms," Journal of the Mathematical Society of Japan, vol. 47, no. 4, pp. 789-793, 1995.
[5] Z. Leśniak, "On the structure of Brouwer homeomorphisms embeddable in a flow," Abstract and Applied Analysis, vol. 2012, Article ID 248413, 8 pages, 2012.

[6] M. C. Zdun and Z. Leśniak, "On iteration groups of singularityfree homeomorphisms of the plane," Annales Mathematicae Silesianae, vol. 8, pp. 203-210, 1994.

[7] N. P. Bhatia and G. P. Szegö, Stability Theory of Dynamical Systems, Springer, Berlin, Germany, 1970.

[8] Z. Leśniak, "On boundaries of parallelizable regions of flows of free mappings," Abstract and Applied Analysis, vol. 2007, Article ID 31693, 8 pages, 2007.

[9] R. C. McCann, "Planar dynamical systems without critical points," Funkcialaj Ekvacioj, vol. 13, pp. 67-95, 1970.

[10] W. Kaplan, "Regular curve-families filling the plane, I," Duke Mathematical Journal, vol. 7, pp. 154-185, 1940.

[11] Z. Leśniak, "On a decomposition of the plane for a flow of free mappings," Publicationes Mathematicae Debrecen, vol. 75, no. 12, pp. 191-202, 2009.

[12] F. Béguin and F. Le Roux, "Ensemble oscillant d'un homéomorphisme de Brouwer, homéomorphismes de Reeb," Bulletin de la Société Mathématique de France, vol. 131, no. 2, pp. 149-210, 2003.

[13] F. Le Roux, "Classes de conjugaison des flots du plan topologiquement équivalents au flot de Reeb," Comptes Rendus de l'Académie des Sciences I: Mathematics, vol. 328, no. 1, pp. 4550, 1999.

[14] S. Matsumoto, "A characterization of the standard Reeb flow," Hokkaido Mathematical Journal, vol. 42, no. 1, pp. 69-80, 2013. 


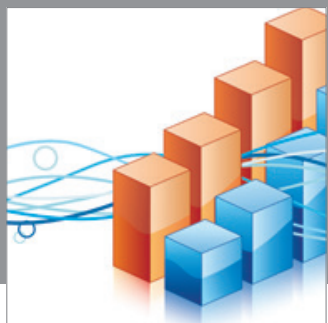

Advances in

Operations Research

mansans

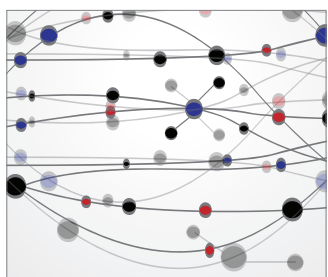

The Scientific World Journal
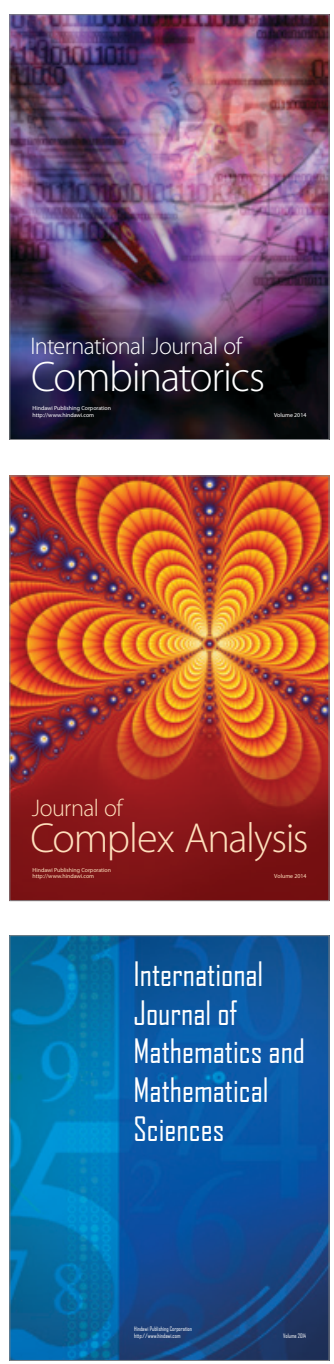
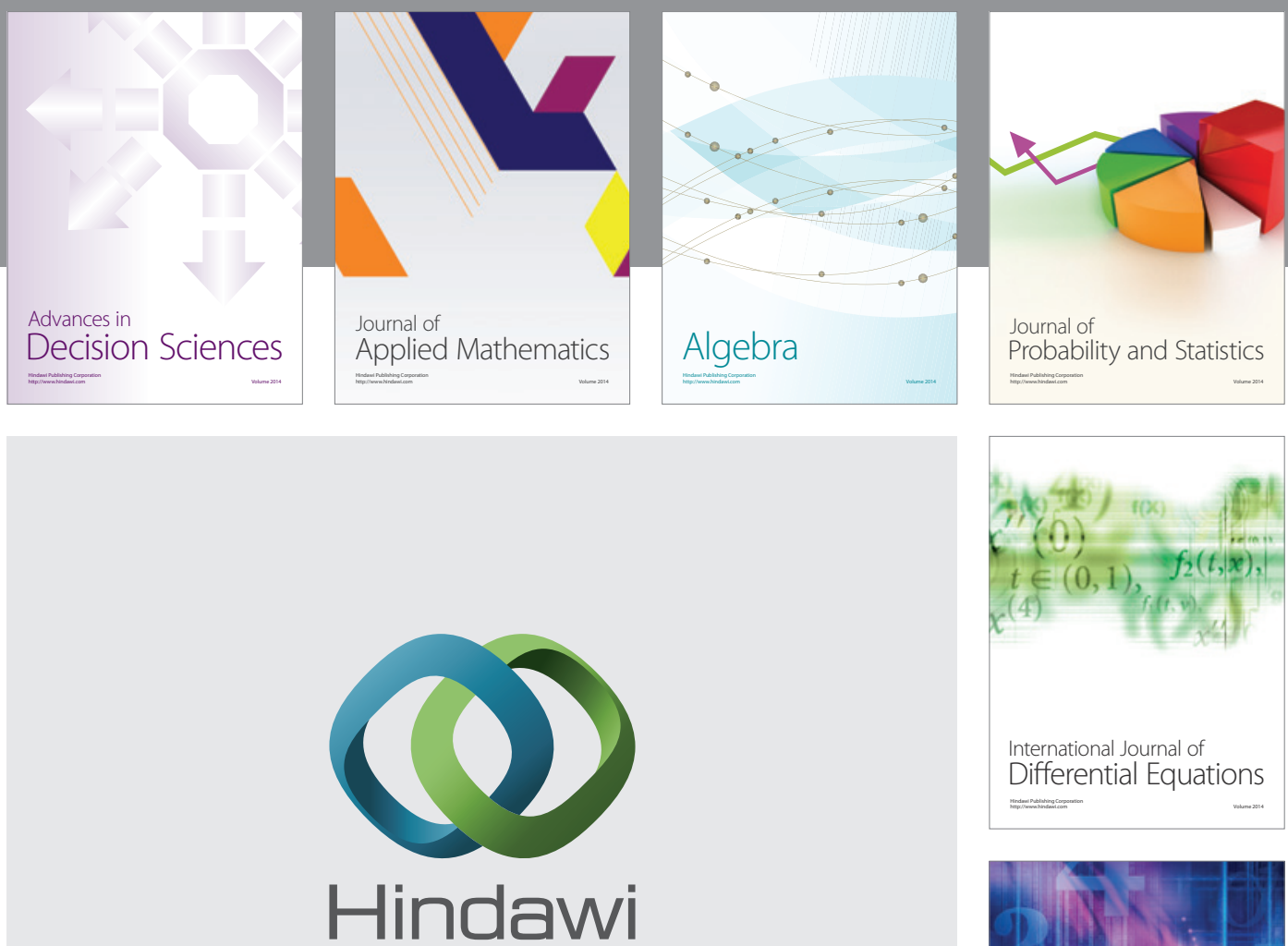

Submit your manuscripts at http://www.hindawi.com
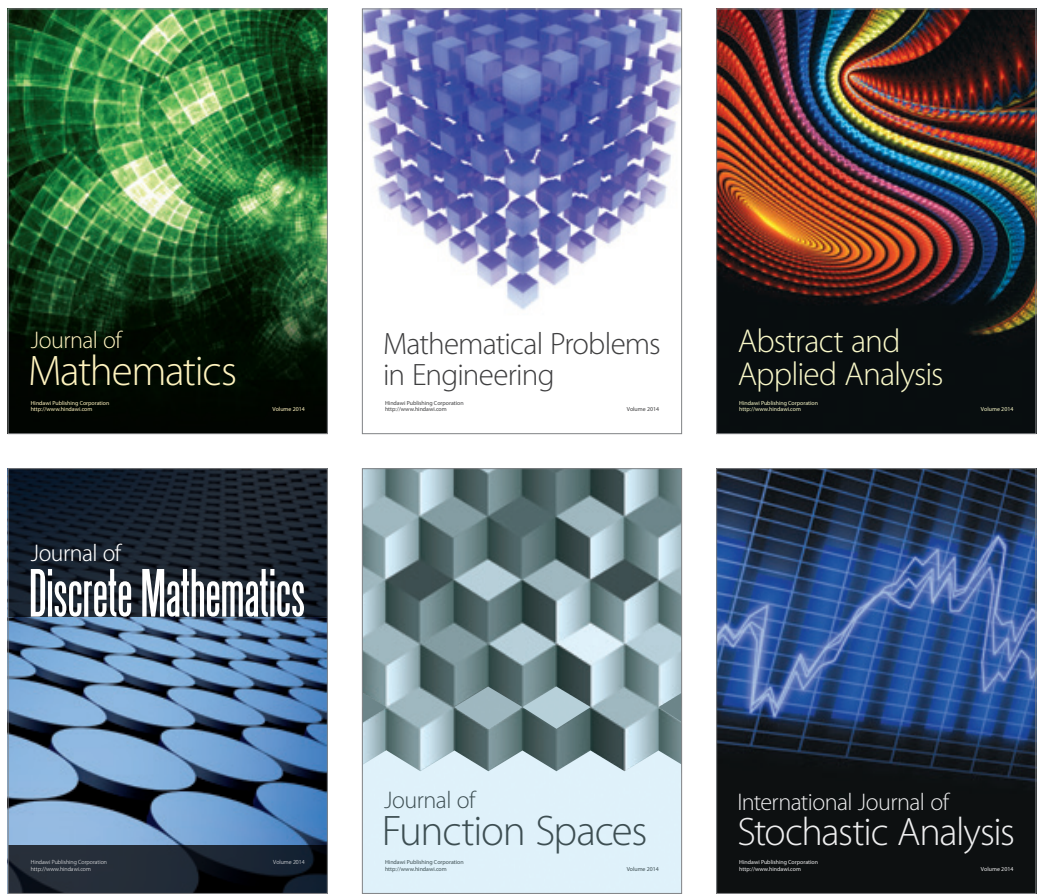

Journal of

Function Spaces

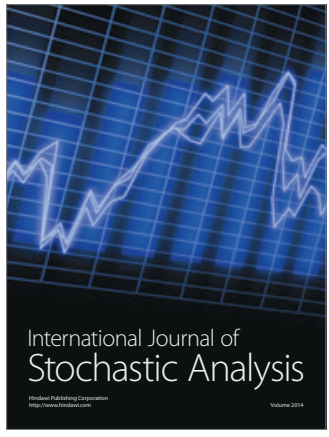

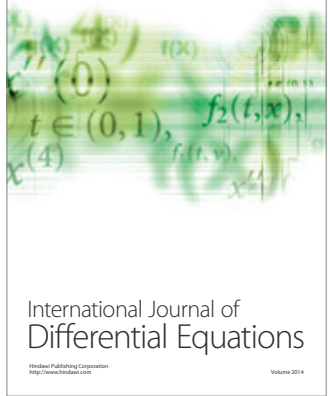
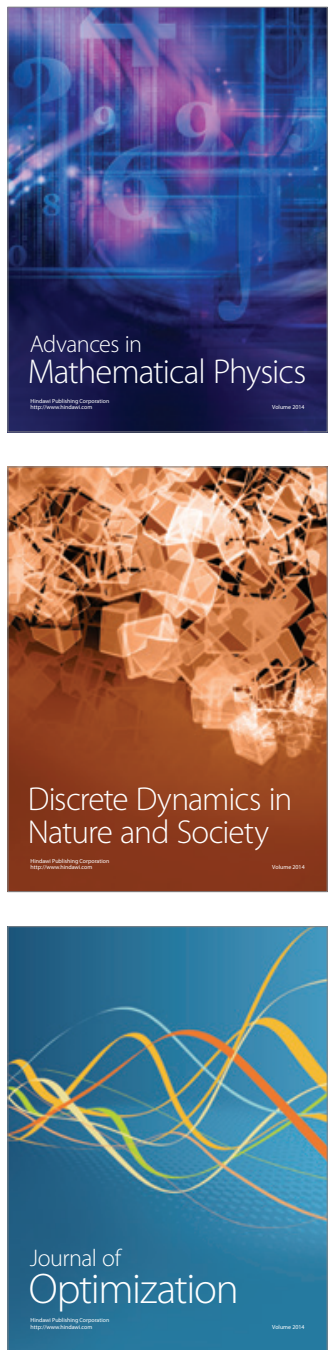